\title{
Reciprocating Engine Step Load Response in Islanded Power Generation
}

\author{
Yun Tian, Rudie Crous \\ Power Group, WorleyParsons Ltd., Perth, Australia \\ Email: tianyun2004@gmail.com
}

Received April, 2013

\begin{abstract}
The step load response of reciprocating engines is one of the key characteristics when considering its application in medium to large scale stationary power generation especially with islanded generation. This paper discusses the impacts of power frequency deviation on the generators and electrical equipment in the network and presents the relationship between step load capabilities and generator operating parameters. For a power plant consisting of a number of generators both step load and power output requirements must be satisfied. An analysis method is proposed to facilitate the development of an operation strategy which can meet both step load and power demand requirements in the full load range. Typical reciprocating engine step load curves are used to demonstrate the analysis method and the results are further optimised for lower operational cost. This analysis method provides a general approach to operation strategy of large reciprocating engines used in islanded power generation.
\end{abstract}

Keywords: Step Load; Islanded Power Generation; Reciprocating Engine; Power Frequency Control

\section{Introduction}

Reciprocating engines have been widely used as power sources or mechanical drives in both transportation (including on-road and sea-borne) and stationary power generation (including base load generation, emergency or backup power generation). In recent years, reciprocating engines have seen its applications expanded to medium and large scale stationary power generation markets, including both islanded generation and commercial grid connected power supply.

Reciprocating engine technology evolved at a fast pace in the last decade, and this enabled larger capacity, better efficiency, dual fuel capability, and higher availability engines to be available to the market. Large reciprocating engines used for stationary power generation are typically in the $6 \mathrm{MW}$ to $18 \mathrm{MW}$ capacity range per unit. These engine generators typically operate at medium speed (500 rpm to $1000 \mathrm{rpm}$, depending on capacity and grid frequency) due to trade-offs between performance and operational life / maintenance interval requirements for base load or continuous operation. Lower speed engines $(<500 \mathrm{rpm})$, feature low dynamic performance and massive physical size and weight, due to low power intensity (output per unit of volume/displacement) related to the low speed, are less attractive than medium speed engines in stationary power generation applications.

These advantages have made large reciprocating en- gines especially attractive for islanded power generation where power quality requirements are not as stringent as those for commercial power grid or utility power generation. However, reciprocating engines still have to meet project requirements and in some cases, be suitable for interconnection to a commercial power grid. These requirements include emissions, availability, frequency control, heat rate, etc. Among these requirements, frequency control or engine's step load response is often neglected in manufacturer's performance guarantee but could prove to be a major issue for some applications.

Frequency response to load changes for gas turbines and steam turbines are well known thanks to their dominance in large scale power generation for more than a century. With a short history in medium to large scale stationary power generation, reciprocating engine's performance on frequency control is a relatively new area for power engineers, planners and plant owner, although it is an important issue for both grid connected and islanded power generations.

Frequency control is implemented through generator governor control, assisted inherently by the power system's moment of inertia, frequency-dependent load response and ancillary service. For commercial grid connected application, any load/supply imbalance and subsequent frequency control requirements could be shared by the large generators connected to the network, especially gas turbines, together with contracted ancillary 
service [1]. The large capacity and inertia of a power grid can often absorb most imbalances easily.

Compared to gas and steam turbines, reciprocating engines has lower inertia to help overcome the initial frequency/speed change, mainly due to its low speed. For reciprocating engines operating in power island mode where no gas/steam turbine or ancillary service is available, frequency control depends totally on the reciprocating engine's capability thus network stability becomes crucial to islanded generations that have large load variations.

This paper first discussed frequency requirements from both power network and consumer equipment perspectives, followed by the relationship between operation parameters and step load capability under design frequency change limits for large capacity reciprocating engines. The focus of this paper is to provide an analysis method to identify reciprocating engine power plant operating conditions based on individual engine generator step load capability, fuel type, number of operating generators and power demand to satisfy frequency control requirements. Typical reciprocating engine step load curves have been used in the analysis and the results are discussed. The analysis method also includes optimisation of operating conditions to minimise fuel cost.

\section{Frequency Requirements for Power Network and Island Power Generation}

Frequency control is very important for the safe operation of a power network, mainly because of the strong relationship between frequency change, supply/demand imbalance and generator protection. Frequency changes have the following impacts to the equipment in the network:

- Generators connected to the power grid: When a power demand/supply imbalance occurs, grid frequency and generator speed will change as a response to this imbalance because of time lag of mechanical power input. Generators are designed to operating within a certain speed range with automatic protection to disconnect them from the grid when frequency change is more than a predetermined value.

- $\quad$ Rotating equipment driven by electric motors which are essentially reverse of generators: There is a fixed relationship between frequency, number of poles in the induction motor field and the rotation speed. Changes in grid frequency will change the rotating equipment speed therefore its work load.

- Some electronic equipment or electric timers depend on the grid frequency as a time reference.

- Harmonic filters. Component overstress may happen when impedance change significantly due to frequency deviation.
Commercial power grid frequency deviation is usually limited to a very small range by regulations in many countries in the world. For example, in Australia, the 'normal operating frequency band' as provided by the National Electricity Rules is set at $49.85 \mathrm{~Hz}$ to $50.15 \mathrm{~Hz}$, or $50 \pm 0.15 \mathrm{~Hz}$ [2][3][4]. This frequency band was set despite some studies on grid frequency finding that the actual tolerance for frequency change are in general much wider than the regulated limits from the perspectives of generators, grid stability, timer control and consumer equipment [5].

For islanded power generation, the frequency deviation depends on contractual requirements, technology selection and the possibility of future interconnection with a commercial power grid. Considerations should be given to generator speed control range for stability of the power grid and motor maximum allowed speed for the protection of mechanical driven equipment (motor, gear, belt, etc.).

Motors built in accordance to National Electrical Manufacturers' Association (NEMA, US) standards are designed to operate successfully at rated load and at rated voltage with a variation in the frequency of up to $5 \%$ above or below the rated frequency (MG1-12.42) [6]. Large motors used in industrial process are often controlled by Variable Speed Drives (VSDs) which are insensitive to frequency deviation.

Frequency deviation will affect AC generator and transformer performance. To avoid the overheating of AC generator core laminations due to flux density, International Electrotechnical Commission (IEC) standard IEC 60034-1 requires industrial AC generators to be designed to operate within a maximum ratio between voltage and frequency (the volts-per-hertz ratio). The frequency deviation limits for temporary operation with reduced performance (Zone B) is between $+3 \%$ and $-5 \%$ [7].

AC Generators are diving by prime movers, e.g., turbines or reciprocating engines, whose speed controls the power line frequency. The speed of the prime movers must avoid resonance and is controlled by the speed governor. The maximum frequency change corresponding to the governor response by changing the air or fuel inlet vane/gate, or the governor droop, is typically set to $5 \%$ by manufacturers. Generator protection system will disconnect the generator from network when frequency deviation is outside of the allowed range, this may cause instability of the power network as the power imbalance may deteriorate. This is especially the case for an under frequency situation.

A tighter range of frequency control limits can protect equipment in the network and reduce the risk of system collapse. It is also recognized that for island power generation, wider limits for voltage and frequency changes are appropriate to accommodate the effects of a weak 
distribution network. The purpose of this paper is to establish an analysis method which can be used for step load increase and step load decrease at any frequency change limits. To facilitate the analysis, we assume $\pm 4 \%$ as the targeted frequency change limit.

\section{Reciprocating Engine Step Load Response}

Step loads are instantaneous load changes in a power network. For an island power network, there are basically three types of power imbalance that can occur instantaneously:

- $\quad$ Failure of one generator which causes insufficient power supply;

- Failure of an electrical component in the electrical network which causes either oversupply or undersupply of power depending on the location of the equipment;

- $\quad$ Large consumer load start-up or trip which causes either sudden increase or decrease in power demand.

Generators respond to the imbalance of power demand/supply by increasing or decreasing power output, and in extreme situations, disconnecting from the network. The response will not be instantaneous mainly due to time lag in the engine or turbine's mechanical components which control the fuel and air input. During this transit time, the prime mover's speed will increase or decrease as the first response to the load imbalance and the maximum speed or frequency change is a function of its starting point and system inertia, i.e., mass and speed of the rotor of engine generators.

As discussed above, reciprocating engines have lower inertia compared to gas or steam turbines. The speed/ frequency change of reciprocating engines depends on engine design, load imbalance, engine load factor (ratio between initial load and generator net capacity) and fuel type (gas/diesel). The maximum step load allowed for a specific reciprocating engine generator without exceeding frequency limits can be determined by its load factor and fuel type. Reciprocating engine generators step load response has the following characteristics:

- $\quad$ Allowed step loads (increase or decrease) for a certain frequency limit vary with load factor and fuel type;

- $\quad$ Dual fuel reciprocating engines operating in diesel mode has a higher step load capability than in gas mode;

- $\quad$ Reciprocating engines have a different capability for step load increase and decrease. Engine generators typically allow equal or larger step load decrease than step load increase for the same speed change.

- $\quad$ For a step load increase, an engine's step load capability increases with the increase of load factor until it reaches its maximum value, then starts to decrease when load factor continues increase.

The analysis method proposed in this paper focuses on step load increase, because step load increase causes more stress to the engine generator rotor and requires a narrower operation range than step load decrease. To meet the step load increase requirement, operating generators must maintain some level of spinning reserve above actual power demand. A typical performance data for step load increase with maximum speed change of 4\% is presented in the Table 1 and illustrated in Figure 1. This step load increase curve, not associated with any specific engine generator model, is used as a start point to demonstrate the analysis approach described in the following sections. The same approach can also be used for step load decrease analysis.

\section{Analysis Approach for Power Plant Operation Under Frequency Change Limits}

The step load curves provided individual engine generator's step load capability under given speed/frequency change limit. Maximum step loads can be conveniently established for different power demand for each individual generator using the curves. For a power plant consisting of a number of reciprocating engine generators, the operation scenarios become more complicated than a single generator. For a given power demand, the operating engine generators must satisfy the following two contradicting requirements:

- The net power output of all operating generators must meet the power demand. The number of generators in operation will be determined based on the power demand and should be operating at a load factor as high as possible to obtain better efficiencies for the same fuel type;

- $\quad$ Power plant must have sufficient spinning reserve to cope with a step load increase. The spinning reserve of all the operating generators shall be capable of coping with a step load increase and maintain frequency change under the limit.

Table 1. Typical reciprocating engine step load increase capability.

\begin{tabular}{|c|c|c|c|c|c|c|c|c|c|c|}
\hline \multicolumn{11}{|c|}{ Diesel Mode } \\
\hline $\begin{array}{c}\text { Load } \\
\text { Factor }\end{array}$ & $\%$ & $0 \%$ & $50 \%$ & $50 \%$ & $70 \%$ & $100 \%$ & & & & \\
\hline $\begin{array}{c}\text { Step } \\
\text { Load }\end{array}$ & $\%$ & $15 \%$ & $15 \%$ & $30 \%$ & $30 \%$ & $0 \%$ & & & & \\
\hline \multicolumn{11}{|c|}{ Gas Mode } \\
\hline $\begin{array}{c}\text { Load } \\
\text { Factor }\end{array}$ & $\%$ & $0 \%$ & $20 \%$ & $20 \%$ & $40 \%$ & $40 \%$ & $60 \%$ & $60 \%$ & $80 \%$ & $100 \%$ \\
\hline $\begin{array}{c}\text { Step } \\
\text { Load }\end{array}$ & $\%$ & $0 \%$ & $0 \%$ & $10 \%$ & $10 \%$ & $15 \%$ & $15 \%$ & $5 \%$ & $5 \%$ & $0 \%$ \\
\hline
\end{tabular}




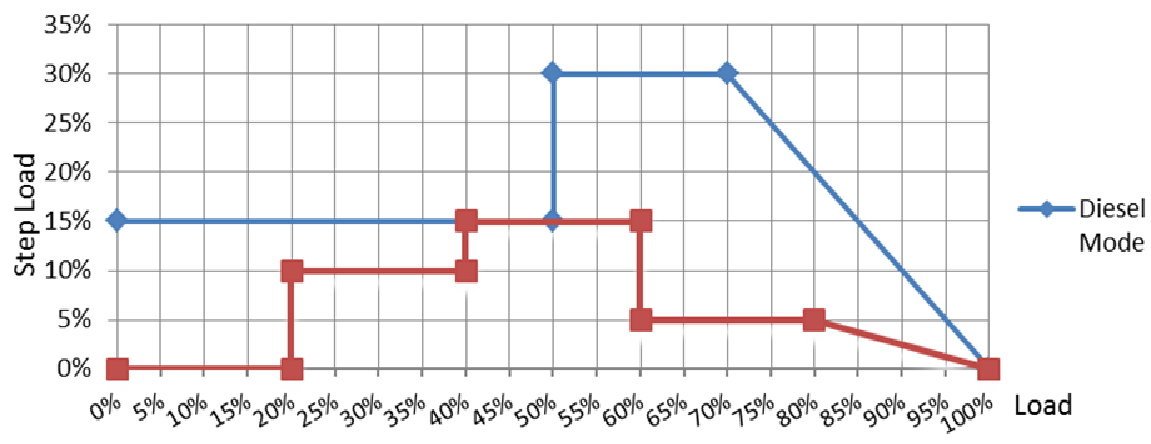

Figure 1. Typical reciprocating engine step load increase curve.

During the engineering phase, for example, the frequency limit and maximum step load, instantaneous power demand from starting of the largest motor in a process plant are typically defined in the Basis of Design document. Power plant configuration will be determined based on a number of criteria including both technical and commercial requirements. The technical factors are mainly contracted maximum power demand, plant availability, emission limits and step load capability.

\section{Analysis Approach for Power Plant Operation Under Frequency Change Limits}

The step load curves provided individual engine generator's step load capability under given speed/frequency change limit. Maximum step loads can be conveniently established for different power demand for each individual generator using the curves. For a power plant consisting of a number of reciprocating engine generators, the operation scenarios become more complicated than a single generator. For a given power demand, the operating engine generators must satisfy the following two contradicting requirements:

The net power output of all operating generators must meet the power demand. The number of generators in operation will be determined based on the power demand and should be operating at a load factor as high as possible to obtain better efficiencies for the same fuel type;

Power plant must have sufficient spinning reserve to cope with a step load increase. The spinning reserve of all the operating generators shall be capable of coping with a step load increase and maintain frequency change under the limit.

During the engineering phase, for example, the frequency limit and maximum step load, instantaneous power demand from starting of the largest motor in a process plant are typically defined in the Basis of Design document. Power plant configuration will be determined based on a number of criteria including both technical and commercial requirements. The technical factors are mainly contracted maximum power demand, plant avail- ability, emission limits and step load capability.

Once the power plant configuration is determined, power plant operation scenarios can be established for the whole range of power demand to satisfy the above technical requirements. The maximum step load allowed for a power plant is a function of power demand, generator step load curve for giving frequency limit, fuel type and generator net capacity. Among these parameters, the power demand, number of operating generators and fuel type are variables once plant configuration is fixed. To determine the operating conditions, a two-step analysis method is proposed in this paper. The first step is to establish all possible operating conditions for the whole range of power demand, which can be optimized in the second step based on fuel cost. To simplify the analysis, all generators are assumed to be identical, but the method can also be applied to plants consist of generators in different capacity or technology.

In the first step, the relationship between power demand, step load and load factors need to be established. Load factor is the link between power demand and step load capability in step load curves and can be determined by:

$$
L F=\frac{P D}{n \times C P}
$$

where

$L F$ - Load factor

$n$ - Number of operating generators

$P D$ - Power demand

$C P$ - Individual generator's net power output

Equation (1) can also be written as

$$
P D=(n \times C P) \times L F=k \times L F
$$

For a specific reciprocating engine, the relationship between individual generator's load factor and step load capacity for giving speed/frequency limit can be expressed as

$$
S L G=f(L F, F L)
$$

where

$S L G$ - step load capability of individual generator, as a 
percentage of the generator's design net power output, and

\section{FL - fuel type}

Generator net power output using different fuels may be slightly different even at the same load factor; therefore net full load power output for the specific fuel should be used in the conversion to absolute values in MW. To simplify the analysis, the slight difference in net power output and heat rate for different fuel types at same load factor is ignored.

For the whole power plant, plant step load capability for the frequency limit can be calculated by

$$
S L P=S L E \times(n \times C P)=k \times S L G
$$

For a giving step load, or design step load, $S L D$, the power plant step load capability under this operating condition must satisfy:

$$
S L P \geq S L D
$$

Combining equations from (1) to (5), leads to the expression for the operating conditions to satisfy step load requirement for a certain power demand and frequency limit:

$$
k \times f\left(\frac{P D}{R}, E L\right) \geq S E D
$$

or

$$
(n \times C P) \times f\left(\frac{P Q}{n \times C P}, F l\right) \geq S E D
$$

where $n$ is restrained by:

$$
n \leq N
$$

where $N$ is the number of installed generators in the power plant.

The power output of operating generators shall also satisfy power demand and maximum generator capacity requirements:

$$
P D \leq(n \times C P)
$$

for step load decrease, or

$$
(P D+S L D) \leq(n \times C P)
$$

for step load increase.

For step load increase, because the instantaneous step load is larger than the actual demand increase after stabilisation, the power output will meet power demand if equation 10 is satisfied.

Equation (7) shows that for a given power plant configuration and design step load, the only variables are fuel types $\mathrm{Fl}$, number of operating generators $\mathrm{n}$ and power demand PD. The step load curves of generator are fixed when generators are selected, and $\mathrm{n}$ is constrained by equations (8), (9) or (10), depending on step load decrease or increase. This means power plant step load capability could either meet design step load for a certain range of power demands, or may not be able to satisfy the whole range of power demand, depending on the generator's step load curve characteristics and fuel type.

For a power plant consisting of 5 x10MW generators and based on the typical generator step load curves shown in Figure 1, the relationship between step load capability and power demand can be illustrated in the Figure 2, assuming design step load of 6.0MW and all 5 generators are in operation $(n=5)$.

Figure 2 shows the initial load (or initial power demand) range that satisfies the design step load under frequency change limit is from $20 \mathrm{MW}$ to $30 \mathrm{MW}$ in gas mode, or from $0 \mathrm{MW}$ to $44 \mathrm{MW}$ in diesel mode.

The relationship shown in Figure 2 gives the possible operational conditions for 5 operating generators, but for a certain power demand, this may not be the best operation mode. The load factors, hence generator efficiencies, may be lower than operating in modes, such as using 4 operating generators, which may also be able to cope with the same design step load.

To establish an optimal power generation solution for the whole range of power demand, it is necessary to determine the heat rate or efficiency of all the operating conditions that can satisfy both step load requirements and power demand requirement. In order to do this, all the power plant's initial load ranges that can meet these requirements at different operating scenarios are explored and the relationship between heat rate and power demand established.

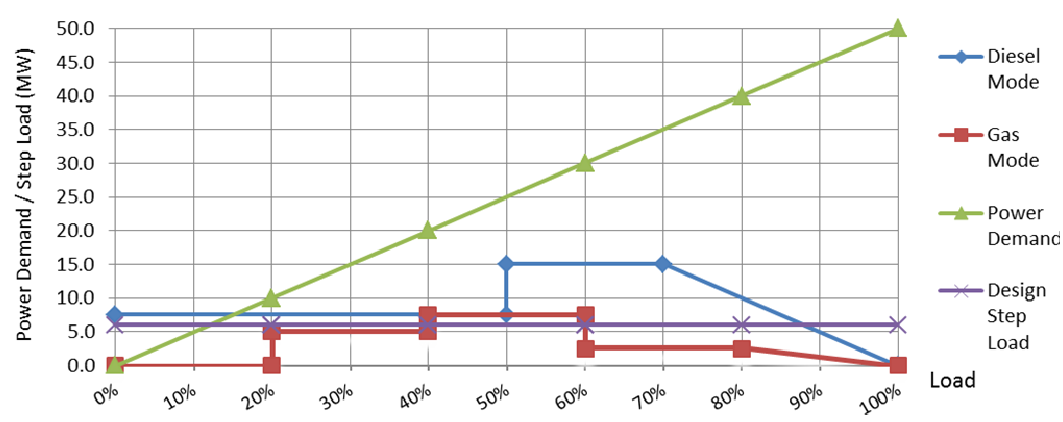

Figure 2. Relationship between step load, load factor and power demand. 
From results of the first step, initial loads satisfying step load requirements under certain number of generators are obtained. Repeating the first step for all the other operating scenarios with different number of operating generators and fuel types, the relationship between power demand and initial loads are determined. Table 2 below summarizes the operating conditions that meet step load requirement using different numbers of operating generators and fuel types. The operating conditions that cannot meet the step load requirement for the whole load range are not shown in the Table 2 including 1 generator in either gas or diesel mode, and 2 or 3 generators in gas mode.

The initial loads are plotted against power demand in Figure 3.

Figure 3 shows that for each power demand, there are often more than one operation mode meeting all the requirements, therefore optimisation is required to determine the best operation mode for each power demand.

Step 2 is to optimise the operation mode based on heat rate and fuel price. A reciprocating engine generator's heat rate is a function of fuel type and load factors. Fuel cost $(\$ / M W H)$ is the product of heat rate and fuel price (\$/GJ). Based on the results from step 1, using typical heat rate curves for reciprocating engines at 10MW ca- pacity range, and assuming fuel price of $6 \$ / G J$ (gas) and $20 \$ / G J$ (diesel), the heat rate and fuel cost can be established against the power demand, as shown in Figure 4.

Figure 4 shows that although reciprocating engine plant in diesel mode dominates the suitable operation conditions, the fuel costs for power plant operating in gas mode are much lower than that in diesel mode, albeit at lower load factors than in diesel mode, for example, a power plant operating in gas mode with 4 or 5 generators has lower fuel costs that it operating in diesel mode with 3 or 4 generators. However, optimal operating modes should also consider other factors, e.g., maintenance cost, which is related to the total engine firing hours and fuel types.

\section{Results and Discussion}

The results show that suitable reciprocating engine power plant operating modes for a giving step load depends on a number of factors. While increasing the number of operating generators may increase the initial load range, this will also reduce load factors which will increase the heat rate, i.e., reduce efficiency. Reciprocating engine power plant operating in gas mode is limited to a small range of initial loads with a higher number of operating generators; while in diesel mode, the power plant has a

Table 2. Generator operating scenarios for step load.

\begin{tabular}{|c|c|c|c|c|c|}
\hline \multirow{2}{*}{$\begin{array}{l}\text { Operating } \\
\text { Conditions } \\
2 \text { Engines }\end{array}$} & \multirow{2}{*}{$\begin{array}{c}\begin{array}{c}\text { Each Engine Step } \\
\text { Load Increase (MW) }\end{array} \\
3.00\end{array}$} & \multirow{2}{*}{$\begin{array}{c}\begin{array}{c}\text { Engine Load } \\
\text { Change (\%) }\end{array} \\
30.00 \% \\
\end{array}$} & \multirow{2}{*}{$\begin{array}{c}\text { Engine Mode and Initial Load Factor } \\
\text { Requirement for maximum } \mathbf{4 \%} \\
\text { Speed Change }\end{array}$} & \multicolumn{2}{|c|}{$\begin{array}{c}\text { Required Plant Initial Load } \\
\text { for maximum } 4 \% \text { Speed Change } \\
(\text { Min/Max, MW) }\end{array}$} \\
\hline & & & & 10.0 & 14.0 \\
\hline 3 Engines & 2.00 & $20.00 \%$ & Diesel Mode: $50.0 \%$ - $80.0 \%$ load factor & 15.0 & 24.0 \\
\hline \multirow{2}{*}{4 Engines } & \multirow{2}{*}{1.50} & \multirow{2}{*}{$15.00 \%$} & Diesel Mode: $0 \%$ - 85\% load factor & 0.0 & 34.0 \\
\hline & & & Gas Mode: $40 \%$ - $60 \%$ load factor & 16.0 & 24.0 \\
\hline \multirow{2}{*}{5 Engines } & \multirow{2}{*}{1.20} & \multirow{2}{*}{$12.00 \%$} & Diesel Mode: $0 \%$ - $88 \%$ load factor & 0.0 & 44.0 \\
\hline & & & Gas Mode: $40 \%$ - $60 \%$ load factor & 20.0 & 30.0 \\
\hline
\end{tabular}

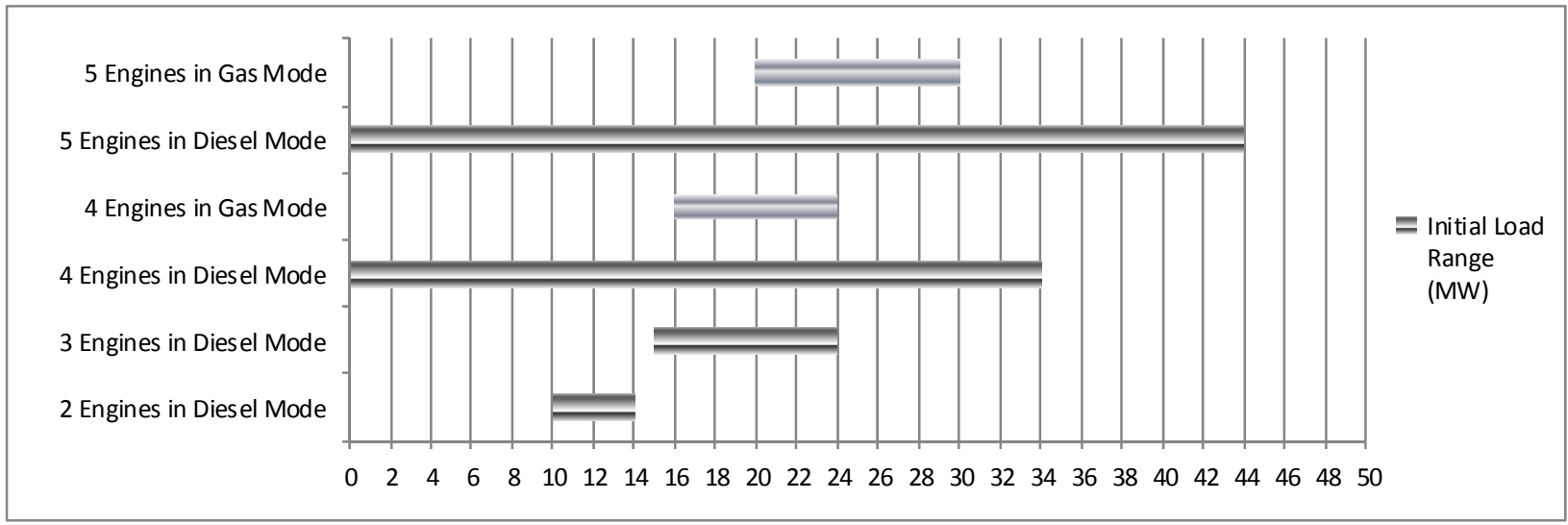

Figure 3. Initial load range at different operating scenarios. 
Fuel Cost at Step Load Capable Operating Conditions

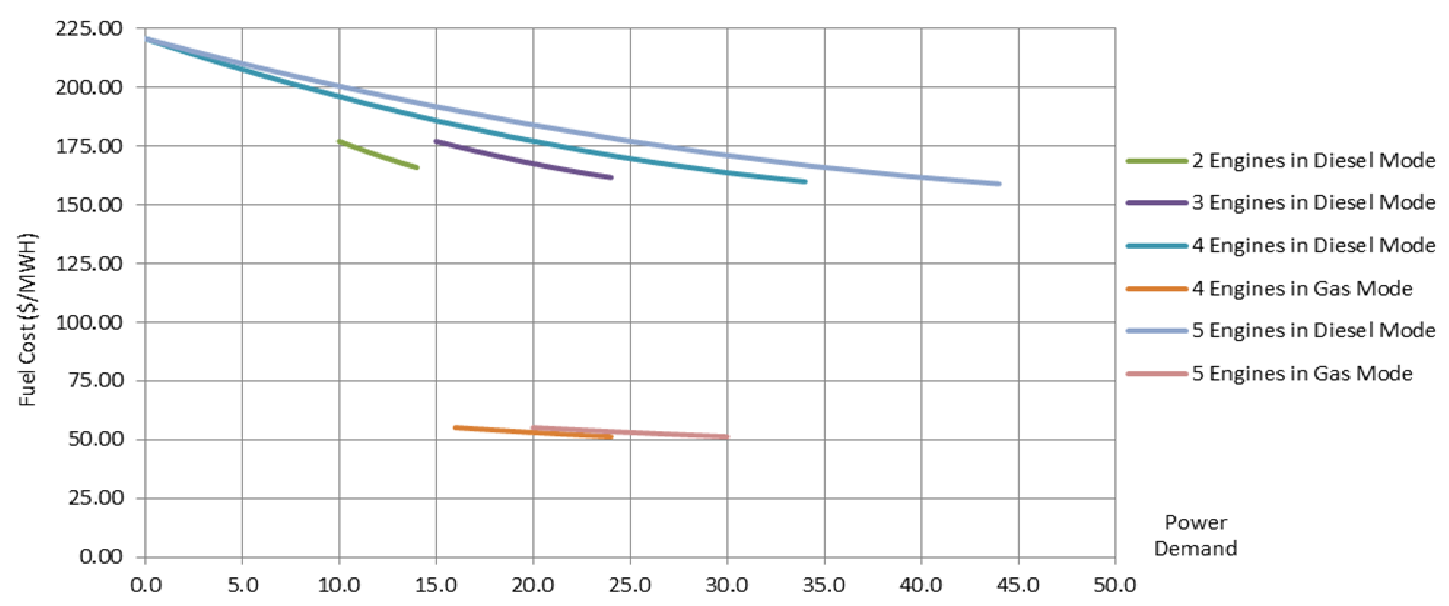

Figure 4. Fuel cost at step load capable operation conditions.

larger initial load range with less operating generators. For a certain power demand, less operating generators can improve load factors, reduce emissions and maintenance levels therefore diesel mode is more favourable from step load response and frequency control perspective. The analysis results also show that when both fuels are suitable for a certain power demand, the fuel costs of power plant in diesel mode are much higher than in gas mode based on the above fuel price assumptions.

\section{Conclusions}

Step load capability and frequency change represents one of the key issues for reciprocating engine's application in medium to large scale stationary power generation. This paper reviews the frequency impacts to generators and consumer equipment in the network and the influence factors to step load response are discussed.

An analysis method is proposed to establish the operation conditions for a given step load and to allow for optimisation of the operating conditions. The proposed power plant step load capability analysis method includes two steps. The first step is to identify all the operating modes that can satisfy both step load and power output requirements for the whole range of power demand. The second step investigates the fuel cost for all the suitable operating modes and allows for optimisation of the operation the whole range of power demand.

The analysis method can be applied to any reciprocating engine step load curves and will help create operation strategies for the whole range of power demand to meet both step load frequency control and power supply requirements. The optimisation of the operation conditions can reduce the fuel cost while maintaining network stability. The results can greatly reduce the complexity of frequency control in manual control procedure prior to the occurrence of step loads, or could be programmed into generator automatic control settings with an early speed change detection system to control the frequency changes in design limits.

\section{REFERENCES}

[1] M. A. Hanley, "Frequency Instability Problems in North American Interconnections,” National Energy Technology Laboratory (United States), 2011.

[2] Power Quality Manager, "Supply Standards: Electricity Supply Standard,” Essential Energy, 2011.

[3] National Electricity Rules (Australia), Version 55 (2013).

[4] Power System Frequency and Time Deviation Monitoring Report - Reference Guide, Australia Energy Market Operator, 2011.

[5] National Electricity Code Administrator, "Reliability Panel Frequency Standards Consulting Paper,” 1998.

[6] B. J. Kirby, etc., "Frequency Control Concerns In The North American Electric Power System," Oak Ridge National Laboratory (United States), 2002.

[7] International Electrotechnical Commission (IEC) Standard 60034-1, "Rotating Electrical Machines - Part 1: Rating and Performance”. 